\title{
NEO-LORENTZIAN RELATIVITY
}

\author{
S. J. PROKHOVNIK
}

(received 5 April 1964, revised 5 September 1964)

\begin{abstract}
Our present view of the universe suggests that the set of mutually receding galaxies may provide a natural substratum for the propagation of light. It is shown that this assumption leads to a consistent derivation and interpretation of special relativity, along the lines envisaged by Lorentz but requiring also the employment of Einstein's measurement definitions. The time-dilatation and Fitzgerald contraction effects emerge as intelligible consequences of this approach, and their interaction with an associated anisotropy effect produces the relativity of simultaneity, the reciprocity phenomenon and the results described by Einstein's principles; the approach provides a definitive resolution of the "clock paradox" within the framework of Special Relativity.
\end{abstract}

\section{Assumptions}

The concept of a cosmological substratum, linked with Mach's principle, has been revived in recent years with the recognition that the space of our universe has field properties determined by the density and distribution of matter and affecting, in turn, the behaviour of matter and light. The possible nature of such a substratum has been investigated by Bastin and Kilmister [1] and its implications for gravitational theory have been developed, by Sciama [2], Bastin [3] and Surdin [4], among others.

It is suggested that the nature of the cosmological substratum is closely linked with the kinematic mode of light propagation in our universe. Our present view of the universe suggests that its "fundamental particles", the galaxies, are distributed homogeneously (apart from local irregularities) and that they are receding from one another according to Hubble's Law. Hence, we can imagine a family of mutually receding fundamental observers whose viewpoint of the universe and of the laws of nature (including Newton's laws) is identical. The implication that the law of light propagation is also the same for all fundamental observers is consistent with an hypothesis, proposed by McCrea [5], that a light-ray passes every 
fundamental observer in its path with velocity $c$. The hypothesis provides a simple interpretation of the Doppler redshift effect and, as has been shown by the author [6], it makes fundamental observers Lorentz-equivalent if their mutual recession is assumed uniform. However, its most important consequence for our present purposes, is that it implies the existence of a specific substratum delineated by the family of fundamental observers. Relative to these observers the propagation of light is isotropic but for all others this is not the case. We distinguish therefore between the fundamental observers (and particles) who are stationary relative to the substratum and "moving" observers (and bodies) who are in motion relative to it. In this way motion assumes an absolute significance which is linked moreover with an immediate observable distinction - the recession of the galaxies, as displayed by the Doppler red-shift, will not appear isotropic to moving observers.

The model derives from and resembles many others. In particular it appear at first sight very similar to the Kinematic Relativity of Milne [7] who also distinguishes between fundamental and subsidiary (that is, moving relative to the substratum) Galilean frames in a uniformly-expanding universe. However, Milne's approach, in common with most others, lacks a specific mode of light-propagation; hence in order to preserve the relativistic equivalence of fundamental observers, Milne introduces two scales of time related by a logarithmic formula. In our case, as is seen in [6], this formula (expressing a measurement relationship) is a direct result of the light hypothesis and it is the elaboration of this assumption which gives the model its interest and advantages. In particular, we are interested here in the consequence that for the moving (or subsidiary) observers, the propagation of light (and more generally of all forms of energy) is not isotropic. The resulting anisotropy leads to a number of physical and measurement effects whose interaction leads in turn to the relativistic equivalence of all Galilean frames.

Builder [8] appears to have been the first to recognise the relativistic consequences of an anisotropy effect. We are concerned here with the mathematical development of this approach (but in a cosmological context) and the demonstration that it provides an intelligible and self-consistent expression of Special Relativity.

We consider therefore as our basic assumptions:

I. In any given locality of the universe there exists a reference frame, $I_{S}$, (with a fundamental observer, $S$, at its origin) relative to which the propagation of energy is isotropic. Since Newton's laws of motion hold for all fundamental observers, $I_{S}$ is an inertial reference frame. It follows that a system $I_{A}$ in the same locality, associated with a reference frame in uniform motion relative to $I_{S}$, is also an inertial system, since a body at rest or in 
uniform motion in $I_{S}$ is equally so in $I_{A} . I_{S}$ will be considered as the basic system for the locality.

II. The movement of a body relative to $I_{S}$ is associated with a single physical effect, the contraction of its length in the direction of motion. Specifically for a body moving with velocity $u_{A}$ in $I_{S}$, its length in the direction of motion is proportional to $\left(1-u_{A}^{2} / c^{2}\right)^{\frac{1}{2}}$, a factor which will be denoted by $\beta_{A}^{-1}$.

The synchronisation of clocks and the measurement conventions proposed by Einstein will be referred to as "Einstein measures". These have become refined through the years and we will employ them in the lightsignal measurement form given by Synge [9].

\section{The time-dilatation effect}

By using $I_{S}$ as a basic reference frame we can relate the corresponding time-intervals and measurements of observers in relative motion.

Following Builder [8] we imagine that $S$ has a number of identical clocks each consisting of a rigid rod, of length $l$, with mirrors at each end to reflect a beam of light to and fro along the length of the rod. Let the time be measured in terms of a unit which is the interval between successive light reflections on one of the mirrors. For a rod stationary in $I_{S}$ the unit $\hat{t}_{S}$ is given by

$$
\hat{t}_{S}=2 l / c
$$

Now imagine that $S$ gives $A$ one of these clocks when $A$ passes him with uniform velocity $u_{A}$ and that $A$ carries it at an angle $\theta$ (according to $S$ ) to the direction of motion. The rod is contracted in the direction of motion only, so that if the length of the moving rod is now $l^{\prime}$ (according to $S$ ), then

whence

$$
\left(\beta_{A} l^{\prime} \cos \theta\right)^{2}+\left(l^{\prime} \sin \theta\right)^{2}=l^{2}
$$

$$
l^{\prime}=\left(l / \beta_{A}\right)\left[1-\left(u_{A} \sin \theta\right)^{2} / c^{2}\right]^{-\frac{1}{2}}
$$

Further, since the rod is moving in $I_{S}$, the velocity of a light-ray along the rod and relative to it will be different for the two directions, say $c_{1}$ and $c_{2}$ where

$$
\begin{aligned}
& c_{1}=\left(c^{2}-u_{A}^{2} \sin ^{2} \theta\right)^{\frac{1}{2}-u_{A}} \cos \theta, \\
& c_{2}=\left(c^{2}-u_{A}^{2} \sin ^{2} \theta\right)^{\frac{1}{2}}+u_{A} \cos \theta .
\end{aligned}
$$

The corresponding unit of time in $I_{A}$ is then

$$
\hat{t}_{A}=\left(l^{\prime} / c_{1}\right)+\left(l^{\prime} / c_{2}\right)=(2 l / c) \beta_{A},
$$

the result being independent of $\theta$. 
We note that if $\theta_{A}$ is $A^{\prime}$ 's measure of the angle the rod makes with the direction of motion, then $\theta$ and $\theta_{A}$ are related by

$$
\tan \theta=\beta_{A} \tan \theta_{A}
$$

in consequence of $A$ 's measuring stick being contracted according to (2.2).

From (2.1) and (2.4) we obtain

$$
\hat{t}_{A}=\beta_{A} \hat{t}_{S}
$$

Similarly for an observer $B$ moving with velocity $u_{B}$ in $I_{S}$,

$$
\hat{t}_{B}=\beta_{B} \hat{t}_{S}
$$

Hence if $A$ and $B$ are moving along a common straight line in $I_{S}$ and measure their times $t_{A}$ and $t_{B}$ from the instant of $A$ and $B$ 's spatial coincidence, then these are related through the corresponding $I_{S}$ time $t_{S}$ by

$$
t_{S}=\beta_{A} t_{A}=\beta_{B} t_{B}
$$

The result can be considered as applying not only to clocks but to all phenomena involving electromagnetic impulses and hence, following Builder $[10]$, to all natural phenomena.

\section{The anisotropy and associated effects}

Consider first how Einstein's synchronism procedure applies to two observers $A$ and $A^{\prime}$, stationary in $I_{A}$ and employing similar clocks. Let $d$ be the $I_{S}$ distance separating $A$ and $A^{\prime}$, and $\theta$ the angle that the direction of $A A^{\prime}$ makes with the direction of $u_{A}$. Let $A$ transmit a light ray at $t_{A}^{1}$ so that it reflects $A^{\prime \prime}$ s clock and returns to $A$ at $t_{A}^{8}$, according to his clock; these times correspond to $\beta_{A} t_{A}^{1}$ and $\beta_{A} t_{A}^{3}$ in $I_{S}$ time. Let the $I_{S}$ time of reflection of $A^{\prime \prime}$ s clock be $t_{S}^{r}=\beta_{A} t_{A}^{r}$. The light-ray travels from $A$ to $A^{\prime}$ and back again with the respective velocities $c_{1}$ and $c_{2}$ as given by (2.3). Hence

$$
\beta_{A}\left(t_{A}^{r}-t_{A}^{1}\right)=d / c_{1}
$$

and

$$
\beta_{A}\left(t_{A}^{3}-t_{A}^{r}\right)=d / c_{2}
$$

so that

$$
t_{A}^{r}-t_{A}^{m}=\left(\beta_{A} u_{A} / c^{2}\right) d \cos \theta
$$

where $t_{A}^{m}=\frac{1}{2}\left(t_{A}^{1}+t_{A}^{3}\right)$, is $A$ 's Einstein measure of the time of the light-ray's reflection at $A^{\prime}$.

To satisfy the synchronism criterion, $A$ requires that the light-ray 
should reflect the reading $t_{A}^{m}$ on $A^{\prime \prime}$ 's clock. However, according to $S$ the reflection takes place simultaneously with the reading $t_{A}^{r}$ on $A$ 's clock, so the difference between the two readings results in different views of simultaneity in $I_{S}$ and $I_{A}$. Further, since the difference is essentially an anisotropy effect and depends on the magnitude of $u_{A}$, it follows that clocks $A$ and $A^{\prime}$ synchronous in $I_{A}$ will not appear so in any other system $I_{B}$ unless the direction of $A A^{\prime}$ is normal to the directions of both $u_{A}$ and $u_{B}$. It is seen that the synchronism is reciprocal since the angles that $\overrightarrow{A A^{\prime}}$ and $\overrightarrow{A^{\prime} A}$ make with the direction of $u_{A}$ are supplementary, and that it is also transitive on account of the group nature under addition of $\left(\beta_{A} u_{A} / c^{2}\right) d \cos \theta$, remembering that $d \cos \theta$ is the projection of $A A^{\prime}$ in the direction of $u_{A}$. Thus Einstein's synchronism definition is self-consistent with respect to any inertial system in spite of the inequality of the light paths involved for all systems except $I_{S}$.

Adding (3.1) and (3.2) leads to

$$
c\left(t_{A}^{3}-t_{A}^{1}\right)=2 \beta_{A} d\left[1-\left(u_{A} \sin \theta\right)^{2} / c^{2}\right]^{\frac{1}{2}}
$$

Hence, $A$ 's Einstein measure, $d_{A}$, of the space interval $A A^{\prime}$ is related to the $I_{S}$ measure $d$ by

$$
d_{A}=\frac{c}{2}\left(t_{A}^{3}-t_{A}^{1}\right)=\beta_{A} d\left[1-\left(u_{A} \sin \theta\right)^{2} / c^{2}\right]^{\frac{1}{2}}
$$

Comparing this relation with (2.2), it is seen that the space interval $A A^{\prime}$ will have the same measure whether $A$ uses a (contracted) measuring rod or the Einstein light-signal convention. In consequence $A$ 's measure of the velocity of light in $I_{A}$, as given by the ratio of $2 d_{A}$ to $\left(t_{A}^{3}-t_{A}^{1}\right)$, will always be $c$ irrespective of the direction or location of $A^{\prime}$. And this result will also hold for the one way clocking of a light-ray using two previously-synchronised clocks, since the synchronisation procedure sets the clocks on the assumption that light traverses a space interval in half the time it would take for an out-and-return journey over the interval.

The result applies for all inertial systems and also in respect to light from outside sources whether in relative motion or not. Thus Einstein's light principle emerges as an unambiguous consequence of our assumptions - in the sense that the measure of the velocity of light is the same with respect to all inertial systems. The result confirms the claims of Builder [8].

\section{The Lorentz transformation}

Consider two observers, $A$ and $B$, moving with uniform velocities $u_{A}$ and $u_{B}\left(u_{B}>u_{A}\right)$ respectively, and in the same straight line (the path of a light-ray in $I_{S}$ ) relative to the system $I_{S} . A$ and $B$ measure their time with 
similar clocks from the instant of their spatial co-incidence, and this instant is also taken as the zero $I_{S}$ time according to a third similar clock associated with an observer $S$ so that (2.7) applies. We note that according to $S$, the relative velocity of $A$ and $B$ is $\left(u_{B}-u_{A}\right)$, and the $I_{S}$ space interval separating $A$ and $B$, at time $t_{S}$, is $\left(u_{B}-u_{A}\right) t_{S}$.

We will take the point, in $I_{S}$, of $A$ 's and $B$ 's spatial co-incidence as the origin of a reference system for $I_{S}, A$ 's location as the origin for $I_{A}$ and $B$ 's location as the origin for $I_{B}$. We will refer to the straight line joining these three origins, and its extension in either direction, as the common $x$-axis of $I_{S}, I_{A}$ and $I_{B}$, where the direction $A$ to $B$ is taken as the positive direction of this axis.

Let $A$ 's Einstein measure of $B$ 's relative velocity be denoted by $v$. To determine this $A$ requires the measure, $s_{A}$, of the space interval, $A B$, at two separate times. For one of these he can use $s_{A}=0$ when $t_{A}=0$. For the second he must employ a light-ray observation from which he can obtain $B$ 's co-ordinates in $I_{A}$, viz. $\left(x_{A B}, t_{A}^{m}\right)$, since $B$ lies on the $x$-axis. Thus $s_{A}=x_{A B}$ when $t_{A}=t_{A}^{m}$. These measures are related to the corresponding $I_{S}$ measures, $s$ and $t_{S}^{r}$, by (2.7), (3.3) and (3.5) with $\theta=0$, so that

where

$$
t_{A}^{r}-t_{A}^{m}=\beta_{A} s u_{A} / c^{2}, \text { and } x_{A B}=\beta_{A} s \text {; }
$$

Then

$$
s=\left(u_{B}-u_{A}\right) t_{S}^{r}=\left(u_{B}-u_{A}\right) \beta_{A} t_{A}^{r} .
$$

$$
v=\frac{x_{A B}-0}{t_{A}^{m}-0}=\frac{\beta_{A}^{2} t_{A}^{r}\left(u_{B}-u_{A}\right)}{t_{A}^{r}-\beta_{A}^{2} t_{A}^{r}\left(u_{B}-u_{A}\right) u_{A} / c^{2}}=\frac{u_{B}-u_{A}}{1-u_{B} u_{A} / c^{2}} .
$$

It is easily verified that $B$ will have the same measure as $A$ of their mutual relative velocity.

Consider, further, an event on a body $E$ whose motion in $I_{S}$ is arbitrary. Since the location of a given event can always be considered co-planar with the locations of $A$ and $B$, it will be convenient to assume a $y$-axis, through each origin, in this common plane and normal to the common $x$-axis. The corresponding $z$-axes are then normal to the plane. We may denote the $I_{S}$ co-ordinates of the event by $\left(x_{S}, y_{S}, z_{S}, t_{S}^{r}\right)$, where $t_{S}^{r}$ is identical to the Einstein measure, $t_{S}^{m}$, by any observer $S$. $A$ 's $I_{A}$ co-ordinates based on his Einstein measures of the event, may be denoted by $\left(x_{A}, y_{A}\right.$, $\left.z_{A}, t_{A}^{m}\right)$, and $B^{\prime}$ s corresponding $I_{B}$ co-ordinates by $\left(x_{B}, y_{B}, z_{B}, t_{B}^{m}\right)$. Thus if $\rho$ is the $I_{S}$ space-interval, separating $A$ and $E$, at $t_{S}^{r} ; \theta$ the angle, according to $S$, that $A E$ makes with the $x$-axis; and if $\rho_{A}, t_{m}^{A}$ and $\theta_{A}$ are the corresponding measures according to $A$, then within each inertial system, separately,

$$
x_{S}=u_{A} t_{S}^{r}+\rho \cos \theta, \quad y_{S}=\rho \sin \theta, \quad z_{S}=0
$$

and 


$$
\begin{aligned}
x_{A} & =\rho_{A} \cos \theta_{A}, & y_{A} & =\rho_{A} \sin \theta_{A}, \quad z_{A}=0 ; \\
& =\beta_{A} \rho \cos \theta, & & =\rho \sin \theta,
\end{aligned}
$$

on applying (2.5) and (3.5). Hence

$$
x_{A}=\beta_{A}\left(x_{S}-u_{A} t_{S}^{r}\right), \quad y_{A}=y_{S}, \quad z_{A}=z_{S}
$$

Also applying (2.7) and (4.2) in (3.3), we obtain the rest of the transformation (4.3), viz.

$$
t_{A}^{m}=t_{A}^{r}-\left(\beta_{A} u_{A} / c^{2}\right) \rho \cos \theta=\beta_{A}\left(t_{S}^{r}-u_{A} x_{S} / c^{2}\right)
$$

By relating the $I_{B}$ and $I_{S}$ measurements we would obtain similarly

$$
\begin{aligned}
x_{B} & =\beta_{B}\left(x_{S}-u_{B} t_{S}^{\tau}\right), \quad y_{B}=y_{S}, \quad z_{B}=z_{S} ; \\
t_{B}^{m} & =\beta_{B}\left(t_{S}^{r}-u_{B} x_{S} / c^{2}\right)
\end{aligned}
$$

Eliminating $x_{S}$ and $t_{S}^{r}$ from (4.3) and (4.4) and using (4.1) then yields,

$$
\begin{aligned}
x_{A} & =\beta_{A B}\left(x_{B}+v t_{B}^{m}\right), \quad y_{A}=y_{B}, \quad z_{A}=z_{B} \\
t_{A}^{m} & =\beta_{A B}\left(t_{B}^{m}+v x_{B} / c^{2}\right),
\end{aligned}
$$

where

$$
v=\frac{u_{B}-u_{A}}{1-u_{B} u_{A} / c^{2}}, \quad \beta_{A B}=\left(1-v^{2} / c^{2}\right)^{-\frac{1}{2}}
$$

The relationship (4.5) is generally known as the Lorentz transformation and it may be generalised to apply to the Einstein measures of an event of any pair of non-accelerated observers in $I_{S}$. The transformation makes the Maxwell equations invariant and on combining it with Newton's first and second laws (conservation and rate of change of momentum), which hold for all inertial systems, we obtain a system of mechanics which again has the invariance property. Thus Einstein's relativity principle is also a consequence of our assumptions.

\section{The operation of relativistic effects}

The measurements related by the Lorentz transformation embody the interaction of the time-dilatation and anisotropy effects. Hence, to appreciate, within our context, the basis of the reciprocity and other aspects of the transformation, it is necessary to disentangle the contributions of the two effects. For instance the relavistic Doppler effect is immediately intelligible from our approach, for if the frequency of a light-ray is $\gamma$ according to $S$, then on account of (2.6) its frequencies according to $A$ and $B$ are related by 


$$
\begin{aligned}
\frac{\gamma_{A}}{\gamma_{B}} & =\frac{\beta_{A}\left(1-u_{A} / c\right) \gamma}{\beta_{B}\left(1-u_{B} / c\right) \gamma} \\
& =\left(\frac{1+v / c}{1-v / c}\right)^{\frac{1}{2}}, \quad \text { using (4.1). }
\end{aligned}
$$

It is of interest to consider the operation of the various effects in the context of an out and return journey since it leads to different measures of time by two observers who have been in uniform relative motion, presenting a phenomenon which appears to contradict the reciprocity of observations between such observers.

Consider then, an observer $A$ stationary in $I_{A}$ moving with velocity $u_{A}$ in a straight line relative to $I_{S}$. Consider also a "traveller" $B$, leaving $A$ with velocity $u_{1}$ relative to $I_{S}$ along the same straight line, proceeding with this velocity until he is at a distance $d$ (as measured in $I_{S}$ ) from $A$, then changing his velocity to $u_{2}$ (in $I_{S}$ ) so that he returns to $A$.

Assuming that $A$ and $B$ (as well as any other observers, $S, G$, etc.) are carrying similar clocks, we will denote the time taken for $B$ 's journey according to $B$ 's clock by $T_{B}$ and according to $A$ 's clock by $T_{A} \cdot\left(T_{A}-T_{B}\right)_{A}$ will then denote the difference between these two times, if any, according to $A$ 's standpoint, $\left(T_{A}-T_{B}\right)_{G}$ the corresponding difference according to an observer $G$, stationary in $I_{G}$, etc.

We will assume that the journey is sufficiently long so that the effects of accelerations at the beginning, turning point and end of the journey, can be considered negligible compared to the effects associated with the uniform velocity periods. We will take the direction $A$ to $B$ as the positive direction, so that $d$ is positive, and then it also follows that $u_{2}<u_{A}<u_{1}$, irrespective of the sign of $u_{A}$.

From the absolute viewpoint of an observer $S$, the time taken for the outward journey is $d /\left(u_{1}-u_{A}\right)$, and for the return journey $d /\left(u_{A}-u_{2}\right)$. The corresponding times of $A$ and $B$ depend on their velocities in $I_{S}$, hence, denoting $u_{A} / c$ by $\bar{u}_{A}$, etc.,

$$
\begin{aligned}
\left(T_{A}-T_{B}\right)_{S}= & \frac{d}{u_{1}-u_{A}}\left[\left(1-\bar{u}_{A}^{2}\right)^{\frac{1}{2}}-\left(1-\bar{u}_{1}^{2}\right)^{\frac{1}{2}}\right] \\
& +\frac{d}{u_{A}-u_{2}}\left[\left(1-\bar{u}_{A}^{2}\right)^{\frac{1}{2}}-\left(1-\bar{u}_{2}^{2}\right)^{\frac{1}{2}}\right] \\
> & \frac{d}{2 c^{2}}\left(u_{1}-u_{2}\right)>0,
\end{aligned}
$$

on applying the Binomial theorem and noting that each grouping of the higher powers is also positive tor $u_{2}<u_{A}<u_{1}$. Thus from the $I_{S}$ stand- 
point, the traveller's clock will always be retarded, on returning, relative to the similar and previously synchronised clocks at his base.

Relative to $A, B$ 's out and return velocities will be $v_{1}$ and $-v_{2}$, respectively, where

$$
v_{1}=\frac{u_{1}-u_{A}}{1-\bar{u}_{1} \bar{u}_{A}}, \quad v_{2}=\frac{u_{A}-u_{2}}{1-\bar{u}_{A} \bar{u}_{2}} .
$$

$A$ 's Einstein measure of the distance travelled is $\beta_{A} d$ where $\beta_{A}=\left(1-\bar{u}_{A}^{2}\right)^{-\frac{1}{2}}$, so that

and

$$
\begin{aligned}
& T_{A}=\left(\beta_{A} d / v_{1}\right)+\left(\beta_{A} d / v_{2}\right) \\
& T_{B}=\left(\beta_{A} d / v_{1}\right)\left(1-\bar{v}_{1}^{2}\right)^{\frac{1}{2}}+\left(\beta_{A} d / v_{2}\right)\left(1-\bar{v}_{2}^{2}\right)^{\frac{1}{2}}
\end{aligned}
$$

$$
\begin{aligned}
\left(T_{A}-T_{B}\right)_{A}= & \left(\beta_{A} d / v_{1}\right)\left[1-\left(1-\vec{v}_{1}^{2}\right)^{\frac{1}{2}}\right] \\
& +\left(\beta_{A} d / v_{2}\right)\left[1-\left(1-\bar{v}_{2}^{2}\right)^{\frac{1}{2}}\right] \\
= & \left(T_{A}-T_{B}\right)_{S}
\end{aligned}
$$

on substituting for $v_{1}$ and $v_{2}$.

Now consider an observer $G$, whose $I_{S}$ velocity in the direction of $B$ 's journey is $u_{G}$. According to $G$, stationary in $I_{G}, B$ 's journey takes place along a space interval which is moving with velocity $w_{A}$ relative to $I_{G}$, where

$$
w_{A}=\frac{u_{A}-u_{G}}{1-u_{A} u_{G}} .
$$

Hence the length, $d_{G}$, of this space interval, according to $G$, will be

$$
\begin{aligned}
d_{G} & =\beta_{A} d\left(1-\bar{w}_{A}^{2}\right)^{\frac{1}{2}} \\
& =\frac{d\left(1-\bar{u}_{G}^{2}\right)^{\frac{1}{2}}}{1-\bar{u}_{A} \bar{u}_{G}} .
\end{aligned}
$$

$w_{A}$ is, of course, $A$ 's velocity relative to $G$. $B$ 's out and return velocities, $w_{1}$ and $w_{2}$, relative to $G$, are given by

$$
w_{1}=\frac{u_{1}-u_{G}}{1-\bar{u}_{1} \bar{u}_{G}}, \quad w_{2}=\frac{u_{2}-u_{G}}{1-\bar{u}_{2} \bar{u}_{G}} .
$$

Hence by the same reasoning as before

$$
\begin{aligned}
\left(T_{A}-T_{B}\right)_{G}= & \frac{d_{G}}{w_{1}-w_{A}}\left[\left(1-\bar{w}_{A}^{2}\right)^{\frac{1}{2}}-\left(1-\bar{w}_{1}^{2}\right)^{\frac{1}{2}}\right] \\
& +\frac{d_{G}}{w_{A}-w_{2}}\left[\left(1-\bar{w}_{A}^{2}\right)^{\frac{1}{2}}-\left(1-\bar{w}_{2}^{2}\right)^{\frac{1}{2}}\right] \\
= & \left(T_{A}-T_{B}\right)_{S},
\end{aligned}
$$

on substituting for $w_{A}, w_{1}, w_{2}$ and $d_{G}$. 
Note that the result depends on the discriminating use of the velocities transformation formula. It is not relevant to measurements referred to a single inertial system; so that during the outward journey, the $I_{S}$ relative velocity of $A$ and $B$ is simply $\left(u_{1}-u_{A}\right)$, and the corresponding $I_{G}$ measure is $\left(w_{1}-w_{A}\right)$.

It is seen that $\left(T_{A}-T_{B}\right)$ is always positive and independent of the inertial system with respect to which it is measured. However, we note that it is only the final result which has universal observational validity. The mutual observations of $A$ and and $B$, as well as those of $G$, are inevitably distorted (from the $I_{S}$ viewpoint) during the journey. Only the $I_{S}$ observers can obtain a true "running commentary", yet all commentaries converge, inevitably, to the final result.

How this can be reconciled with the reciprocity aspect tof $A$ 's and $B$ 's observations has caused much concern and bears further examination. As $B$ approaches the turning point, his clock-reading (according to $S$ ) approaches the time $t_{B}^{r}$ where

$$
t_{B}^{r}=\frac{d}{u_{1}-u_{A}}\left(1-\bar{u}_{1}^{2}\right)^{\frac{1}{2}}=d / \beta_{1}\left(u_{1}-u_{A}\right)
$$

Let $A$ 's corresponding clock-reading, according to $S$, be denoted by $t_{A}^{r}$ where

$$
t_{A}^{r}=\frac{d}{u_{1}-u_{A}}\left(1-\bar{u}_{A}^{2}\right)^{\frac{1}{2}}=d / \beta_{A}\left(u_{1}-u_{A}\right)
$$

We note that the values of $t_{A}^{r}$ and $t_{B}^{r}$ are those used in the first part of (5.1).

At this stage $A$ and $B$ 's views of each other's clocks are reciprocal. Thus $A$ 's Einstein-estimate, $t_{A}^{m}$, of the reversal event coincident with $B$ 's clock-reading, $t_{B}^{r}$, is given by applying (3.3), so that

$$
\begin{aligned}
t_{A}^{m} & =t_{A}^{r}-\beta_{A} u_{A} d / c^{2} \\
& =t_{B}^{r}\left(1-\bar{v}_{1}^{2}\right)^{-\frac{1}{2}}=\beta_{A} d / v_{1}
\end{aligned}
$$

on invoking (5.5), (5.6) and (5.2), and noting that

$$
\beta_{1} \beta_{A}\left(1-\bar{u}_{1} \bar{u}_{A}\right)=\left(1-\bar{v}_{1}^{2}\right)^{-\frac{1}{2}}
$$

$B$ 's corresponding estimate, $t_{B}^{m}$, of the event (that is, the appearance of non-uniformity in $A$ 's relative velocity) coincident with $A$ 's clock-reading, $t_{A}^{r}$, is similarly given by (3.3) with $\theta=\pi$ in this case, so that

$$
\begin{aligned}
t_{B}^{m} & =t_{B}^{r}+\beta_{1} u_{1} d / c^{2} \\
& =t_{A}^{r} /\left(1-\bar{v}_{1}^{2}\right)^{\frac{1}{2}}=\beta_{1} d / v_{1}
\end{aligned}
$$

on involking (5.6), (5.5) and (5.2) again. 
(5.7) and (5.8) express the reciprocity phenomenon and demonstrate the role of the anisotropy effect in generating this phenomenon. However, during the reversal period, when $B$ is changing his velocity in $I_{S}$, such reciprocity no longer operates. $A$ 's view remains unaffected, but $B$ 's view is now associated with a succession of inertial frames so that the anisotropy effect associated with his light-signal observations of $A$ undergo a corresponding change. The net effect of $B$ 's reversal (whether carried out slowly or rapidly) on his Einstein observation of the scene at his base is a diminution in the anisotropy effect from $\beta_{1} u_{1} d / c^{2}$, as in (5.8) to $\beta_{2} u_{2} d / c^{2}$, where $\beta_{2}=\left(1-\bar{u}_{2}^{2}\right)^{-\frac{1}{2}}$, since $u_{2}<u_{1}$. And since also $u_{2}<u_{A}$, this now results in $A$ 's clock appearing fast (as is easily confirmed using (3.3) again) compared to $B$ 's according to the latter's Einstein observation. With the resumption of reciprocity during the return journey $A$ 's clock again appears to be losing time but it remains still in advance (according to $B$ ) when $A$ and $B$ are reunited.

Exactly, $B$ 's view of the situation converges to

$$
\begin{aligned}
\left(T_{A}-T_{B}\right)_{B}= & \left(\beta_{1} d / v_{1}\right)\left[\left(1-\vec{v}_{1}^{2}\right)^{\frac{1}{2}}-1\right]+\left(\beta_{1} u_{1} d / c^{2}-\beta_{2} u_{2} d / c^{2}\right) \\
& +\left(\beta_{2} d / v_{2}\right)\left[\left(1-\vec{v}_{2}^{2}\right)^{\frac{1}{2}}-1\right] \\
= & \left(T_{A}-T_{B}\right)_{S}
\end{aligned}
$$

as for all other observers, on making the necessary substitutions. Thus taking into account the change in the anisotropy effect during reversal completely resolves the notorious "clock paradox".

It is seen that the hypothesis of a cosmological substratum for lightpropagation permits of a full and consistent interpretation of the theory and of the absolute effects described by it. It may also provide the key to the physical implications of the theory. One might expect the emergence of physical effects as a result of the anisotropy of electromagnetic and gravitational propagation (and of the resultant fields) relative to moving bodies. A manner in which this may occur has already been outlined by Bastin [3], and the application of his approach by the author [11] suggests that the Fitzgerald contraction may be a primary anisotropy effect resulting from the asymetry of the gravitational and electromagnetic fields relative to a moving system of particles.

In this way the whole theory and all its implications can be considered to rest on the single assumption that the universe provides a natural substratum for the propagation of light. Einstein's principles emerge as intelligible consequences of this approach so it is not surprising that they served as a valid starting point for developing the theory. It is suggested however, that our present picture of the universe provides a better basis for comprehending and applying the theory than was possible in 1905 . 


\section{References}

[1] Bastin, E. W. and Kilmister, C. W., The Concept of Order II Measurements, Proc. Camb. Phil. Soc. 51 (1951), 454.

[2] Sciama, D. W., On the Origin of Inertia, M. N. Roy. Ast. Soc. 113 (1953), 34.

[3] Bastin, J. A., An Extension of the Newtonian Law of Gravitation, Proc. Camb. Phil. Soc. $56(1960), 401$.

[4] Surdin, M., A Note on Time-varying Gravitational Potentials, Proc. Camb. Phil. Soc. $58(1962), 550$.

[5] McCrea, W. H., Some Current Problems in Cosmology, Proc. Math. Soc. Univ. S'ton. 5 (1962), 15.

[6] Prokhovnik, S. J., A Cosmological Model of Light Propagation, Proc. Camb. Phil. Soc. 60 (1964), 265.

[7] Milne, E. A., Kinematic Relativity, Oxford U. P., (1948).

[8] Builder, G., The Constancy of the Velocity of Light, Aust. J. Phys. 11 (1958), 457.

[9] Synge, J. L., Relativity: The Special Theory, Nth. Holland Pub. Co. (1856).

[10] Builder, G., Colloquium on Relativity at Univ, of N.S.W. (1960).

[11] Prokhovnik, S. J., The Case for an Aether, Brit. J. Phil. Sc. 14 (1963), 195.

School of Mathematics,

University of New South Wales 